\title{
Translation and Transliteration
}

Translation of Arabic and Persian texts in English is from standard works the authors of which have been identified. All translations from Urdu and Persian are by the present writer unless otherwise indicated.

Texts from Near Eastern and South Asian languages-Arabic, Persian, and Urdu-have been transliterated using the symbols given below. Hamzah, however, has not generally been transliterated.

Names of contemporary writers who write in English are used as they spell them. Other South Asian names have, however, been transliterated. To avoid unnecessary complication, familiar words and names of people and places in South Asia as well as Arabic names which are often used in the press have been written as they normally appear in books on South Asian studies in the notes and the bibliography. This facilitates referring to them in the literature and library catalogues. To facilitate the reader, names of important people and concepts have been transliterated in the index. Certain words in Arabic and other languages ordinarily used in English and found in dictionaries have not been transliterated. For instance, jihad (jihād), hadith (hadith), madrasah (madrasah), and Qur'an (Qur’ān). Words in direct quotations are written as originally spelled.

\section{Symbols Used for Arabic}

\begin{tabular}{|c|c|c|c|c|}
\hline \multirow{2}{*}{$\begin{array}{c}\text { Letter } \\
\&\end{array}$} & \multirow{2}{*}{$\begin{array}{c}\text { Name } \\
\text { Hamzah }\end{array}$} & \multirow{2}{*}{$\begin{array}{l}\text { IPA } \\
?\end{array}$} & \multicolumn{2}{|c|}{$\begin{array}{c}\text { Modified ALA-LC } \\
\text { (American Library } \\
\text { Association and the } \\
\text { Library of Congress) } \\
\text { Arabic }\end{array}$} \\
\hline & & & & \\
\hline ب & bā' & b & B & b \\
\hline$ت$ & tā' & $\mathrm{t}$ & $\mathrm{T}$ & \\
\hline$ث$ & thā' & $\theta$ & Th & \\
\hline ج & jīm & dz g 3 & J & \\
\hline$\tau$ & ḥā’' & $\hbar$ & 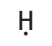 & ḥ \\
\hline$\dot{\tau}$ & khā' & $x$ & $\mathrm{Kh}$ & $\mathrm{kh}$ \\
\hline 2 & Dāl & d & D & $d$ \\
\hline ذ & dhāl & $\partial$ & Dh & $\mathrm{dh}$ \\
\hline J & rā' & $r$ & $R$ & $r$ \\
\hline
\end{tabular}


Symbols Used for Arabic (Continued)

\begin{tabular}{|c|c|c|c|c|}
\hline \multirow{2}{*}{$\begin{array}{l}\text { Letter } \\
j\end{array}$} & \multirow{2}{*}{$\begin{array}{c}\text { Name } \\
\text { zāy }\end{array}$} & \multirow{2}{*}{$\frac{\text { IPA }}{\mathrm{z}}$} & \multicolumn{2}{|c|}{$\begin{array}{c}\text { Modified ALA-LC } \\
\text { (American Library } \\
\text { Association and the } \\
\text { Library of Congress) } \\
\text { Arabic }\end{array}$} \\
\hline & & & Z & z \\
\hline س & $\sin n$ & $\mathrm{~s}$ & $S$ & $\mathrm{~s}$ \\
\hline ش ش & shīn & $\int$ & Sh & sh \\
\hline ص & șuād & $s^{\varsigma}$ & Ș & Ș \\
\hline ض ض & ḍuād & $d^{\varsigma}$ & D & $\stackrel{d}{d}$ \\
\hline$b$ & țā' & $t^{\varsigma}$ & T & $t$ \\
\hline ظ & ẓā' & $\partial^{\varsigma} \sim z^{\varsigma}$ & ? & $z$ \\
\hline$\varepsilon$ & ‘ayn & ؟ & & \\
\hline$\dot{\varepsilon}$ & ghayn & $\gamma$ & Gh & gh \\
\hline ف & fā' & $f$ & $\mathrm{~F}$ & $f$ \\
\hline ق & qāf & $q$ & Q & $q$ \\
\hline ك5 & kāf & k & K & $\mathrm{k}$ \\
\hline J & lām & 1 & L & 1 \\
\hline م & mīm & $\mathrm{m}$ & $M$ & $\mathrm{~m}$ \\
\hline ن & nūn & $\mathrm{n}$ & $\mathrm{N}$ & $n$ \\
\hline 。 & hā' & $\mathrm{h}$ & $\mathrm{H}$ & $\mathrm{h}$ \\
\hline و & wāw & w, u: & W;Ū & $w ; \bar{u}$ \\
\hline ي & yā' & j, i: & Y;ī & $y ; i$ \\
\hline ì & $\begin{array}{c}\text { alif mad- } \\
\text { dah }\end{array}$ & Pa: & $\bar{A}$ & $\bar{a}$ \\
\hline 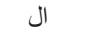 & alif lām & Nil & Al- & al- \\
\hline
\end{tabular}




\section{Symbols used for Persian}

Same as Arabic except the following graphemes in Persian not shared with Arabic.

\begin{tabular}{|c|c|c|c|}
\hline Persian Letter & IPA & \multicolumn{2}{|c|}{$\begin{array}{l}\text { Modified ALA-LC (American } \\
\text { Library Association and } \\
\text { the Library of Congress) }\end{array}$} \\
\hline 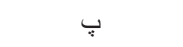 & $/ \mathrm{p} /$ & $P$ & $\mathrm{p}$ \\
\hline ङ & $/ \mathrm{t} J /$ & $\mathrm{Ch}$ & ch \\
\hline j & $|3|$ & $\mathrm{Zh}$ & $\mathrm{zh}$ \\
\hline
\end{tabular}

Symbols used for Urdu

\begin{tabular}{|c|c|c|c|}
\hline j & r & R & $r$ \\
\hline زٔه & th & Ṛh & ṛh \\
\hline$\stackrel{1}{~}$ & $d$ & D & $d$ \\
\hline دُه & dhh & Dh & dhh \\
\hline • & $t$ & T & $t$ \\
\hline ثله & th & Th & thh \\
\hline زهه & th & & \\
\hline
\end{tabular}

/ē /as in Urdu/pēt/=stomach or /lōg/ = people.

/ẽ/ nasalised). 
